\title{
Performance benefits and costs in forced choice perceptual identification in amnesia: Effects of prior exposure and word frequency
}

\author{
Margaret M. Keane \\ Wellesley College, Wellesley, Massachusetts, \\ Boston University School of Medicine, Boston, Massachusetts \\ and Boston VA Healthcare System, Boston, Massachusetts \\ AND \\ Elizabeth Martin and Mieke Verfaellie \\ Boston University School of Medicine, Boston, Massachusetts \\ and Boston VA Healthcare System, Boston, Massachusetts
}

\begin{abstract}
Accuracy in identifying a perceptually degraded word (e.g., stake) can be either enhanced by recent exposure to the same stimulus or reduced by recent exposure to a similar stimulus (e.g., stare). In the present study, we explored the mechanisms underlying these benefits and costs by examining the performance of amnesic and control groups in a forced choice perceptual identification (FCPI) task in which briefly flashed words (that were identical to studied words, similar to studied words, or new) had to be identified, and two response choices were provided that differed from each other by one letter. Control participants showed a performance benefit and cost in FCPI with both high- and low-frequency words. Amnesic participants showed a benefit (but no cost) with high-frequency words and a benefit and a cost with low-frequency words. The benefit/cost pattern with low-frequency words in amnesia was obtained even when the to-be-identified stimulus in the FCPI task was eliminated (Experiment 2), suggesting that this effect was driven by processes operating at the level of the response choices. Our findings suggest that implicit memory effects in FCPI reflect the operation of multiple mechanisms, the relative contributions of which may vary with the frequency of the test stimuli. The results also highlight the need for caution in interpreting results from normal participants in the FCPI task, since those findings may reflect a contribution of explicit memory processes.
\end{abstract}

Accuracy in identifying words and pictures can be affected by recent exposure to the same words or pictures. Importantly, this priming effect can occur even in the absence of explicit memory for the prior encounter with the stimuli: Priming effects are preserved in amnesic participants who have impaired explicit memory for recently encountered stimuli (for a review, see Verfaellie \& Keane, 2001), and priming is often unaffected by experimental manipulations that reduce the availability of explicit memory in control participants (Roediger \& McDermott, 1993). One of the major theoretical goals of memory research over the past several decades has been to understand the nature of the implicit memory processes that support priming effects. Although priming effects may reflect perceptual or conceptual processes (Blaxton, 1989; Roediger \& Blaxton, 1987), much of the theoretical work in the field has been concerned with perceptual-priming effects (Graf \& Ryan, 1990; Moscovitch, Goshen-Gottstein, \& Vriezen, 1994; Schacter, 1990, 1992), and those effects form the basis of the present discussion.
For many years, theoretical and functional accounts of priming focused on the essentially beneficial nature of the phenomenon. Priming was thought to reflect the experience-dependent enhancement or tuning of processes that support accurate identification of words and objects (e.g., Tulving \& Schacter, 1990). Thus, it has been said that "priming in the perception of particular objects or words exists to improve the identification process after an initial occasion" (Roediger, 2003, p. 14). On this view, priming reflects enhanced perceptual discriminability of (or enhanced sensitivity to) recently encountered stimuli. Indeed, the literature is replete with demonstrations of priming-induced enhancements in stimulus identification accuracy.

A growing body of evidence, however, demonstrates that priming may be associated with performance costs as well as benefits. One of the paradigms used most frequently to demonstrate these costs and benefits is the forced choice perceptual identification (FCPI) task introduced by Ratcliff, McKoon, and Verwoerd (1989). The stimuli in this

M. M. Keane, mkeane@wellesley.edu 
paradigm are word pairs that differ from each other by one letter (e.g., case-care). For each pair, either one member or neither member is presented to participants in a study phase. In a subsequent FCPI task, words are flashed very briefly on a computer screen and are followed by two response choices, including the flashed word and its orthographic mate. The participants are asked to choose the response alternative that matches the just-flashed word. In the old condition, the flashed word appears in the prior study phase, so the studied alternative constitutes the correct response. In the lure condition, the orthographic mate of the flashed word appears in the prior study phase, so the studied alternative constitutes the incorrect response. In the unstudied condition, neither the flashed word nor the response alternative appears in the prior study phase, so this condition provides a baseline measure of identification accuracy (see Table 1). If participants are inclined (for whatever reason) to select the response alternative that appeared in the prior study phase, their accuracy in the old condition will be higher than that in the unstudied condition (a performance benefit), and their accuracy in the lure condition will be lower than that in the unstudied condition (a performance cost). Using this paradigm, Ratcliff et al. (1989) demonstrated that control participants show performance benefits and costs of equal magnitude, so that overall accuracy is not enhanced by prior stimulus exposure. These findings challenge the long-standing assumption that implicit memory effects are due to studyinduced changes in sensitivity.

The costs and benefits observed in the FCPI task can be understood in a number of ways. The most influential account of these findings comes from Ratcliff, McKoon, and colleagues (Ratcliff, Allbritton, \& McKoon, 1997; Ratcliff \& McKoon, 1996, 1997; Ratcliff et al., 1989), who argued that priming reflects a bias to identify a current stimulus in accord with a recently encountered stimulus. They have developed a mathematical model (the counter model) that instantiates this bias phenomenon. In this model, every word is associated with a mental representation, referred to as a counter, that accumulates evidence (e.g., from a visual stimulus) about the presence of that word; when the evidence accumulated by a counter exceeds some threshold, word identification occurs (i.e., that word is judged to be present). Recent exposure to a word (e.g., cat) in a study phase increases the attractive force of that word's counter, temporarily increasing the likelihood that that counter will accumulate evidence for that word. Thus, if a person subsequently encounters a perceptually

Table 1

Experimental Conditions in the Forced Choice Perceptual Identification Task

\begin{tabular}{lccc}
\hline \multicolumn{1}{c}{ Phase } & Old & Lure & Unstudied \\
\hline $\begin{array}{l}\text { Study } \\
\text { Test* }\end{array}$ & CASE & CARE & - \\
Flashed word & CASE & CASE & CASE \\
Response choices & CASE CARE & CASE CARE & CASE CARE \\
\hline
\end{tabular}

${ }^{*}$ In the test phase, a word is flashed briefly on the computer screen and is followed by two response choices; participants are asked to select the choice that matches the just-flashed word. degraded (i.e., briefly flashed) word that is similar to cat, the increased attraction exerted by the cat counter makes it more likely that the stimulus will be identified as that word (and that cat will therefore be selected from the two alternatives). If the flashed stimulus is indeed cat, this bias enhances the likelihood of a correct response choice; if the flashed word is car, this bias reduces the chances of a correct response choice.

An alternative explanation of the costs and benefits in the FCPI task rejects the idea that this pattern is due to biased processing of the flashed word and argues, instead, that it reflects (at least in part) a process occurring at the point of selection between the two response alternatives (Bowers, 1999). In support of this view, Bowers demonstrated that performance costs and benefits in the FCPI task were obtained even when the flashed "word" to be identified in the test phase was a string of nonalphabetic symbols, followed by response choices of a studied word and its orthographic mate. In other words, participants' response choices were determined not by their interpretation of the flashed stimulus (which in this case was not even a meaningful item), but simply by their inclination to select a studied over an unstudied word when given such a choice. Such a tendency would enhance accuracy in the old condition (yielding a benefit) and decrease accuracy in the lure condition (yielding a cost). The inclination to select the studied response alternative could be driven by the enhanced processing fluency associated with studied (as compared with nonstudied) stimuli (Jacoby \& Dallas, 1981). In other words, when asked which response alternative matches a just-flashed word, participants may use the relative fluency of the two choices as the basis for their selection (judging that the fluency of the studied alternative must be due to the fact that it matches the just-flashed word). Such an effect would be akin to demonstrations that a variety of decisions (including stimulus preference and judgments of stimulus familiarity, clarity, or pleasantness) may be influenced by the fluency with which stimuli are processed (Reber, Winkielman, \& Schwartz, 1998; Seamon, Brody, \& Kauff, 1983; Whittlesea, Jacoby, \& Girard, 1990). We will return to this idea later in the context of the present paradigm.

A third account of the costs and benefits seen in the FCPI task is that they reflect the operation of explicit, rather than implicit, memory processes. This conclusion follows from a study in our laboratory (Keane, Verfaellie, Gabrieli, \& Wong, 2000) in which amnesic individualswho have marked impairments in explicit memoryperformed an FCPI task modeled on the one described above. In that study, control participants showed performance benefits and costs (as they had in previous studies), but amnesic patients showed a performance benefit alone, with no cost. We interpreted these data as suggesting that the symmetrical cost/benefit pattern in FCPI in normal participants (in our study and in prior studies) is the product of explicit memory mechanisms that are impaired in amnesia. On this view, normal participants' enhanced selection of the studied alternative reflects their tendency to choose words that they remember from the study list. Furthermore, these findings suggest that implicit memory 
processes (preserved in amnesia) operate in accord with a sensitivity mechanism in the FCPI task: Identification accuracy is facilitated by prior exposure to to-be-identified stimuli, and that enhancement is not offset by a performance cost in identification of words that resemble studied stimuli.

Perhaps the most controversial aspect of the conclusions that emerge from our findings in amnesia is the argument that symmetrical costs and benefits observed in control participants in the FCPI task are a by-product of explicit memory processes. For example, although Ratcliff and McKoon $(1996,1997)$ and Bowers (1999) disagree about the precise nature of the implicit memory mechanism underlying the cost/benefit pattern in FCPI, they share the view that those mechanisms operate within the domain of implicit memory.

Masson (2002) offered an alternative account of our findings in amnesia, arguing that this alternative obviates the need to invoke explicit memory to explain the difference in performance between control and amnesic participants in FCPI. Masson noted that sensitivity effects have been observed in normal participants in an FCPI task when the stimuli are low-frequency words (e.g., Bowers, 1999; McKoon \& Ratcliff, 2001; Wagenmakers, Zeelenberg, \& Raaijmakers, 2000). He suggested that the sensitivity effect demonstrated by the amnesic patients in Keane et al. (2000) may have been due to the fact that the stimuli were experienced by the amnesic patients (but not by the control participants) as low-frequency words. (The actual frequency of words used in the study ranged from low to high.) On this view, performance in both control and amnesic groups may be mediated by implicit memory processes, and the distinct pattern of performance across groups may be understood solely with reference to the functional frequency of words in control and amnesic participants (and to the presumably distinct implicit memory mechanisms that are engaged by words of varying frequency).

A closer consideration of the findings in normal cognition, however, reveals that a word frequency account cannot provide a full explanation of our findings in amnesia. The paradigm that has been used to demonstrate sensitivity effects for low-frequency words (described in more detail in the General Discussion section) is a modified version of the FCPI task described above and is designed to measure sensitivity effects in isolation (i.e., with no possible role for bias mechanisms). Although normal participants have shown sensitivity effects for low-frequency words in this modified paradigm (Bowers, 1999; McKoon \& Ratcliff, 2001; Wagenmakers, Zeelenberg, \& Raaijmakers, 2000), it has never been argued that such evidence excludes a role for bias mechanisms in identification of low-frequency words. Notably, in the standard FCPI task, performance costs and benefits are routinely observed for low-frequency stimuli in normal cognition (Wagenmakers, Zeelenberg, \& Raaijmakers, 2000; Zeelenberg, Wagenmakers, \& Raaijmakers, 2002). By contrast, the pattern that we observed in amnesia (Keane et al., 2000) - a benefit in the absence of a cost in the standard FCPI task - points to an exclusive role for sensitivity in the performance of amnesic patients. Although Masson's (2002) word frequency account may be pertinent to the sensitivity effect in amnesia, it cannot explain the absence of a cost/benefit pattern in that group.

Nonetheless, Masson's (2002) argument highlights the possibility that word frequency may have played a role in the pattern of performance that we observed in amnesia. To explore this possibility in the present study, we directly manipulated the frequency of stimulus items in an FCPI task. We selected high- and low-frequency stimuli that were widely separated on the frequency spectrum in order to be confident that the functional frequency of the stimuli for amnesic patients would be lower for the latter than for the former stimuli. Masson's word frequency account of our earlier findings predicts that amnesic patients will show a benefit alone with the low-frequency stimuli but will show a cost/benefit pattern with the high-frequency stimuli. Alternatively, if implicit memory processes operate in accord with a sensitivity mechanism in the FCPI task (as we have hypothesized), amnesic patients should show a benefit alone with both high- and low-frequency stimuli, replicating the results we obtained in our prior study with mixed-frequency word lists.

\section{EXPERIMENT 1A}

In this experiment, we administered two FCPI priming tasks (one with high- and one with low-frequency words) and two forced choice recognition memory tasks (one with high- and one with low-frequency words) to amnesic and control participants. The latter tasks were included to document the explicit memory impairment in the amnesic group in tasks that were as parallel as possible to the priming tasks.

\section{Method}

\section{Participants}

Twelve amnesic individuals ( 7 male, 5 female) with a variety of etiologies, including anoxia $(n=7$, one with a left partial temporal lobectomy), encephalitis $(n=3)$, bithalamic stroke $(n=1)$, and anterior communicating artery aneurysm $(n=1)$, participated in this study. The amnesic group had a mean age of 56.5 years, a mean education of 14.3 years, and a mean verbal IQ score of 102.3, as measured by the Wechsler Adult Intelligence Scale-III (WAIS-III; Wechsler, 1997a). Their attentional abilities were intact, as evidenced by a mean score of 97.5 on the Working Memory Index of the Wechsler Memory Scale-III (WMS-III; Wechsler, 1997b). However, their memory functioning was severely compromised, as indicated by a mean General Memory Index of 59.4, a mean Visual Delay Index of 65.7, and a mean Auditory Delay Index of 62.2 (see Table 2).

The control group included 12 healthy participants ( 4 male, 8 female) who had no prior history of alcohol abuse, neurological illness, or psychiatric disorder. The control group was matched to the amnesic group in terms of age $(M=59.5$ years $)$, education $(M=$ 14.3 years $)$, and WAIS-III verbal IQ $(M=103.17$; all $t \mathrm{~s}<1)$.

\section{Materials}

The critical stimuli were 96 pairs of low-frequency words (mean frequency $=4.29$ per million; range, $0-10$ ) and 96 pairs of highfrequency words (mean frequency $=431$ per million; range, $74-$ 10,790) (Francis \& Kučera, 1982). All the words were four to eight letters in length ( $M=5.0$ for the low-frequency set and 4.6 for the 
Table 2

Neuropsychological Data for Amnesic Participants

\begin{tabular}{|c|c|c|c|c|c|c|c|c|c|c|}
\hline \multirow[b]{2}{*}{ No. } & \multirow[b]{2}{*}{ Expt. } & \multirow[b]{2}{*}{ Gender } & \multirow{2}{*}{$\begin{array}{c}\text { Age at } \\
\text { First Session }\end{array}$} & \multirow[b]{2}{*}{ Edu. } & \multirow[b]{2}{*}{ Etiology } & \multirow{2}{*}{$\begin{array}{c}\text { WAIS-III } \\
\text { VIQ }\end{array}$} & \multicolumn{4}{|c|}{ WMS-III } \\
\hline & & & & & & & GM & VD & $\mathrm{AD}$ & WM \\
\hline 1 & $1 \mathrm{~A}$ & female & 75 & 12 & Anoxia & 107 & 59 & 65 & 64 & 83 \\
\hline 2 & $1 \mathrm{~A}, 1 \mathrm{~B}$ & male & 49 & 14 & Encephalitis & 92 & 45 & 56 & 55 & 85 \\
\hline 3 & $1 \mathrm{~A}, 1 \mathrm{~B}$ & female & 61 & 12 & Encephalitis & 106 & 69 & 68 & 77 & 111 \\
\hline 4 & $1 \mathrm{~A}, 1 \mathrm{~B}$ & male & 75 & 18 & Anoxia & 113 & 75 & 72 & 80 & 102 \\
\hline 5 & $1 \mathrm{~A}, 1 \mathrm{~B}$ & female & 54 & 12 & Anoxia & 83 & 52 & 56 & 55 & 91 \\
\hline 6 & $1 \mathrm{~A}, 1 \mathrm{~B}$ & female & 46 & 14 & Anoxia & 90 & 45 & 53 & 52 & 93 \\
\hline 7 & $1 \mathrm{~A}, 1 \mathrm{~B}$ & male & 48 & 14 & Anoxia & 111 & 59 & 72 & 52 & 96 \\
\hline 8 & $1 \mathrm{~A}, 1 \mathrm{~B}$ & male & 76 & 18 & Encephalitis & 135 & 45 & 53 & 58 & 141 \\
\hline 9 & $1 \mathrm{~A}, 1 \mathrm{~B}$ & male & 63 & 12 & Bilateral thalamic stroke & 84 & 73 & 84 & 67 & 99 \\
\hline 10 & $1 \mathrm{~A}, 1 \mathrm{~B}$ & male & 52 & 17 & Anoxia & 134 & 70 & 75 & 67 & 88 \\
\hline 11 & $1 \mathrm{~A}, 1 \mathrm{~B}$ & male & 42 & 16 & Anoxia + left temporal lobectomy & 86 & 49 & 53 & 52 & 93 \\
\hline 12 & $1 \mathrm{~A}, 1 \mathrm{~B}$ & female & 32 & 12 & $\mathrm{ACoA}$ aneurysm & 87 & 72 & 81 & 67 & 88 \\
\hline 13 & $1 \mathrm{~B}$ & female & 72 & 12 & ACoA aneurysm & 88 & 67 & 84 & 58 & 76 \\
\hline 14 & $1 \mathrm{~B}$ & female & 77 & 12 & ACoA aneurysm & 98 & 73 & 75 & 64 & 96 \\
\hline 15 & $1 \mathrm{~B}$ & female & 65 & 12 & ACoA aneurysm & 93 & 54 & 64 & 58 & 93 \\
\hline 16 & $1 \mathrm{~B}$ & female & 62 & 16 & $\mathrm{ACoA}$ aneurysm & 124 & 82 & 81 & 80 & 102 \\
\hline 17 & $1 \mathrm{~B}$ & female & 66 & 12 & ACoA aneurysm & 93 & 60 & 65 & 58 & 83 \\
\hline
\end{tabular}

Note-WAIS-III, Wechsler Adult Intelligence Scale (3rd ed.; Wechsler, 1997a); WMS-III, Wechsler Memory Scale (3rd ed.; Wechsler, 1997b); No., participant number; Expt., experiment(s) participant completed; Edu., education in years; VIQ, verbal IQ; GM, general memory index; VD, verbal delay index; $\mathrm{AD}$, auditory delay index; WM, working memory index; ACoA, anterior communicating artery.

high-frequency set). Each word in a pair differed from its mate by one letter. Within each word-frequency set, the difference occurred at the beginning of the word for 41 pairs (e.g., pull-full), in the middle of the word for 28 pairs (e.g., change-chance), and at the end of the word for 27 pairs (e.g., duel-duet). For each participant, 96 pairs (48 high frequency and 48 low frequency) were used in the priming tasks, and the remaining 96 pairs ( 48 high frequency and 48 low frequency) were used in the recognition memory tasks. One hundred sixty-eight additional words ( 84 high frequency, $M=$ 221.19 per million; 84 low frequency, $M=3.35$ per million) were selected for use in a preexperimental threshold-setting procedure.

For purposes of the priming tasks, one word in each pair was designated the target word (the word to be flashed in the test phase), and the other the distractor word (the alternative response choice to be paired with the target word in forced choice identification in the test phase). The mean frequency of the target and distractor words did not differ in the high-frequency list $(p>.50)$ or in the lowfrequency list $(p>.50)$. Of the 48 pairs in each word frequency condition, 24 pairs were assigned to the unstudied condition, and 24 to the studied condition. In the unstudied condition, neither word of the pair appeared in the study phase. Of the 24 pairs in the studied condition, 12 were assigned to the old condition, and 12 to the lure condition. In the old condition, the target word from each pair appeared in the study list. In the lure condition, the distractor word from each pair appeared in the study list. In all conditions, the target word was flashed in the identification task (see Table 1).

The recognition memory tasks were designed to parallel the priming tasks as closely as possible. Thus, in each word frequency condition, 24 word pairs were assigned to the unstudied condition (in which neither word of the pair appeared in the study phase) and 24 to the studied condition (in which one word from each pair appeared in the study phase). All 48 pairs appeared in the two-choice recognition test phase. Designation of stimuli to old/lure conditions was not relevant to the recognition memory measure.

For each word frequency condition, complete counterbalancing of items - across the priming and recognition tasks, across the studied and unstudied conditions, and within the studied condition, across the old and lure conditions - required eight test forms. Because the number of participants in each group was not a multiple of eight, we were unable to achieve complete counterbalancing of items across tasks and conditions, but the distribution of forms across conditions was identical for the amnesic and control groups (and was as close as possible to a counterbalanced design).

\section{Procedure}

The experimental procedure was conducted in one session, consisting of two FCPI priming tasks and two recognition memory tasks, each of which included a study phase and a test phase. The priming tasks were administered first, with the order of high- and low-frequency conditions counterbalanced across participants. Each priming task was preceded by a threshold-setting procedure (described below) to determine the appropriate exposure duration for the high- or low-frequency words in the test phase of the priming task to follow. The recognition memory tasks were administered second, with the order of high- and low-frequency conditions counterbalanced across participants. Each participant completed the experiment twice, with alternate test forms, in sessions separated by at least 1 month. (Two control participants were unable to participate in the second session, so the results from the first session were used in place of a mean across sessions.)

All the stimuli were presented in uppercase 36-point Courier font on the screen of a Macintosh computer. The participants were seated approximately $18 \mathrm{in}$. from the screen.

Preexperimental setting of stimulus duration. Prior to each priming task, we determined for each participant the exposure duration that yielded approximately $50 \%$ accuracy in identification of high- (or low-) frequency words without response alternatives. Pilot studies had indicated that this exposure duration would yield above-chance but below-ceiling performance in the forced choice identification task. The participants were asked to identify 60 highor low-frequency words presented at seven different exposure durations ranging from 11 to $117 \mathrm{msec}$. Each word was preceded by a fixation character $(+)$ and was followed immediately by a mask consisting of superimposed $\mathrm{H}$ and $\mathrm{X}$ characters. After an initial estimate of the appropriate exposure duration, four additional 6-word lists were used to verify and adjust the duration necessary to yield approximately $50 \%$ accuracy. That exposure duration was used in the subsequent forced choice identification task. In the control group, the mean exposure duration was $32.3 \mathrm{msec}$ for low-frequency words and $26.9 \mathrm{msec}$ for high-frequency words. For the amnesic group, the mean exposure duration was $42.6 \mathrm{msec}$ for low-frequency words and $35.3 \mathrm{msec}$ for high-frequency words. A two-way mixed factorial 
ANOVA with factors of group and word frequency indicated that the exposure duration was higher for low- than for high-frequency words $[F(1,22)=8.8, p<.01]$ but did not differ between groups $(p>.15)$. There was no group $\times$ frequency interaction $(p>.50)$.

FCPI priming task. Each priming task consisted of a study phase and a test phase. In each study phase, the participants saw a target or distractor word from each of 24 high- or low-frequency word pairs. The words were presented singly on the computer screen for $2 \mathrm{sec}$, and the participants were asked to judge, on a scale of 1 to 5 , how positive or negative the word was. Each word appeared twice, once in the first half and once in the second half of the study list, for a total of 48 trials.

The test phase followed immediately after the study phase. The participants were told that, on each trial, a word would be flashed briefly on the computer screen and would be followed by two response alternatives; they were to choose the alternative that matched the just-flashed word. On each of 48 trials, a target word was presented centrally on the computer screen at the predetermined exposure duration. It was preceded by a fixation $(+)$ and was followed by a mask (consisting of superimposed $\mathrm{H}$ and $\mathrm{X}$ characters) that remained on the screen for approximately $500 \mathrm{msec}$. The response alternatives appeared immediately thereafter and remained on the screen until the participants responded by pressing one of two buttons on the keyboard in front of them. On each trial, one of the response alternatives matched the just-flashed target word, and the other was the distractor mate of the flashed word. On 24 of the trials, neither the flashed word nor its distractor mate had appeared in the prior study list; these trials constituted the unstudied condition and provided a baseline measure of identification performance. The remaining 24 trials constituted the studied (old or lure) conditions: On half of these trials, the flashed word had appeared in the study list (old condition); on these trials, the studied alternative was the correct response choice. On the other half of the trials, the distractor mate of the flashed word had appeared in the prior study list (lure condition); on these trials, therefore, the studied alternative was the incorrect response choice.

Recognition memory task. Each recognition memory task consisted of a study phase and a test phase. In the study phase, a new list of high- or low-frequency words was presented (one word from each of 24 pairs) with the same procedure described above. In the test phase, 48 high- or low-frequency word pairs were presented 1 at a time on the computer screen; half of the pairs included a word seen in the previous study phase, and half did not. (Only the former trials were included in the data analysis.) The participants were asked to choose which of the two words they had seen in the previous study list and to guess if they were unsure. They responded by pressing one of two buttons on the keyboard.

\section{Results}

\section{Baseline Performance in the Perceptual Identification Task}

The goal of the initial threshold-setting procedure was to establish an exposure duration for each participant that would yield above-chance but below-ceiling performance and approximately equal performance across groups and across word frequency conditions in the unstudied (baseline) condition. Such equating is important because the size of study-induced performance changes may be inherently dependent on baseline accuracy (Chapman, Chapman, Curran, \& Miller, 1994). In the control group, accuracy in the unstudied condition was .66 for low-frequency words and .62 for high-frequency words, and in the amnesic group, it was .73 for low-frequency words and .63 for high-frequency words. A two-way mixed factorial ANOVA with factors of group and word frequency indicated that overall accuracy in the unstudied condition did not differ in the amnesic and control groups $(p>.30)$ and that accuracy was higher for low- than for high-frequency words $[F(1,22)=7.4, p<.05]$. The interaction between group and word frequency was not significant $(p>30)$. We will return later to the fact that baseline performance differed in the high- and low-frequency conditions.

\section{FCPI Priming Task}

Low-frequency words. For each participant, we calculated the proportion of words identified correctly in each experimental condition (old, unstudied, lure; see Table 3). These data were submitted to a two-way mixed factorial ANOVA (by participants and by items) with factors of group (control vs. amnesic) and study condition (old vs. unstudied vs. lure). The main effect for group was significant only by items $\left[F_{1}(1,22)=2.8, p>.10 ; F_{2}(1,190)=\right.$ $18.8, p<.001]$, reflecting higher overall accuracy in the amnesic group than in the control group. There was a main effect for study condition $\left[F_{1}(2,44)=39.3, p<\right.$ $\left..001 ; F_{2}(2,380)=90.8, p<.001\right]$, reflecting the benefits and/or costs associated with exposure to items in the prior study list. The interaction between group and study condition was significant $\left[F_{1}(2,44)=4.9, p<.05 ; F_{2}(2,380)=\right.$ $17.2, p<.001]$, indicating that the magnitude of benefits and/or costs differed across the two groups.

We explored this interaction with follow-up ANOVAs to compare performance across groups separately for the benefit and the cost. To examine the benefit, we conducted a two-way mixed factorial ANOVA (by participants and by items) with factors of group and study condition (old vs. unstudied). The main effect for group was not significant $\left[F_{1}(1,22)=0.38, p>.50 ; F_{2}(1,190)=1.6, p>.20\right]$. A performance benefit was indicated by higher accuracy for old words $(.84)$ than for new words $(.70)\left[F_{1}(1,22)=\right.$ $\left.41.1, p<.001 ; F_{2}(1,190)=67.3, p<.001\right]$. The interaction between group and study condition was significant by items and marginally significant by participants $\left[F_{1}(1,22)=3.6, p=.072 ; F_{2}(1,190)=8.7, p<.01\right]$, reflecting the larger benefit in the control group (.19) than in the amnesic group (.10). Analysis of simple effects indicated that the benefit was significant in both the control group $\left[F_{1}(1,22)=34.5, p<.001 ; F_{2}(1,190)=62.3, p<\right.$ $.001]$ and the amnesic group $\left[F_{1}(1,22)=10.2, p<.01\right.$; $\left.F_{2}(1,190)=13.8, p<.001\right]$.

To examine the cost, we conducted a two-way mixed factorial ANOVA (by participants and by items) with factors of group and study condition (unstudied vs. lure). The main effect for group was significant $\left[F_{1}(1,22)=4.8, p<\right.$

Table 3

Mean Proportion Correct in Forced Choice Perceptual Identification Task (Experiment 1A)

\begin{tabular}{|c|c|c|c|c|c|c|c|}
\hline \multirow[b]{3}{*}{ Group } & \multirow[b]{3}{*}{ Frequency } & \multicolumn{6}{|c|}{ Condition } \\
\hline & & \multicolumn{2}{|c|}{ Old } & \multicolumn{2}{|c|}{ Unstudied } & \multicolumn{2}{|c|}{ Lure } \\
\hline & & $M$ & $S E$ & $M$ & $S E$ & $M$ & $S E$ \\
\hline \multirow[t]{2}{*}{ Control $(n=12)$} & Low & .85 & .04 & .66 & .03 & .48 & .05 \\
\hline & High & .83 & .05 & .62 & .04 & .44 & .04 \\
\hline \multirow[t]{2}{*}{ Amnesic $(n=12)$} & Low & .83 & .04 & .73 & .03 & .66 & .05 \\
\hline & High & .72 & .05 & .63 & .04 & .62 & .04 \\
\hline
\end{tabular}


$\left..05 ; F_{2}(1,190)=31.4, p<.001\right]$, indicating that overall accuracy was higher in the amnesic than in the control group. A performance cost was indicated by lower accuracy for lure words $(.57)$ than for unstudied words $(.70)$ $\left[F_{1}(1,22)=24.7, p<.001 ; F_{2}(1,190)=42.2, p<.001\right]$. The interaction between group and study condition was significant $\left[F_{1}(1,22)=4.3, p<.05 ; F_{2}(1,190)=10.9\right.$, $p<.01]$, reflecting the larger cost in the control group (.18) than in the amnesic group (.07). An analysis of simple effects showed that the cost was significant in the control group $\left[F_{1}(1,22)=24.9, p<.001 ; F_{2}(1,190)=\right.$ $48.0, p<.001]$; in the amnesic group, the cost was significant by items and marginally significant by participants $\left[F_{1}(1,22)=4.2, p=.054 ; F_{2}(1,190)=5.1, p<.05\right]$.

In sum, for low-frequency words, there were significant performance benefits and costs in both the amnesic group and the control group, and these benefits and costs were larger in the control group than in the amnesic group.

High-frequency words. The data for high-wordfrequency words (Table 3) were submitted to a two-way mixed factorial ANOVA (by participants and by items) with factors of group (control vs. amnesic) and study condition (old vs. unstudied vs. lure). The main effect for group was not significant $\left[F_{1}(1,22)=0.40, p>.50 ; F_{2}(1,190)=\right.$ $0.80, p>.30]$. There was a main effect for study condition $\left[F_{1}(2,44)=25.9, p<.001 ; F_{2}(2,380)=55.7, p<.001\right]$, reflecting the benefits and/or costs associated with exposure to items in the prior study list. An interaction between group and study condition $\left[F_{1}(2,44)=9.4, p<.001\right.$; $\left.F_{2}(2,380)=20.9, p<.001\right]$ indicated that the magnitude of benefits and/or costs differed across groups.

We explored this interaction with two ANOVAs to compare performance across groups separately for the benefit and the cost. To examine the benefit, we conducted a two-way mixed factorial ANOVA (by participants and by items) with factors of group and study condition (old vs. unstudied). The main effect for group was significant only by items $\left[F_{1}(1,22)=0.70, p>.40 ; F_{2}(1,190)=5.5\right.$, $p<.05]$. A performance benefit was indicated by higher accuracy for old words (.77) than for unstudied words (.62) $\left[F_{1}(1,22)=23.8, p<.001 ; F_{2}(1,190)=41.4, p<\right.$ $.001]$. There was a group $\times$ study condition interaction $\left[F_{1}(1,22)=4.4, p<.05 ; F_{2}(1,190)=6.2, p<.05\right]$, indicating that the benefit was larger in the control group (.21) than in the amnesic group (.09). An analysis of simple effects showed that the benefit was significant in the control group $\left[F_{1}(1,22)=24.3, p<.001 ; F_{2}(1,190)=39.9\right.$, $p<.001]$; in the amnesic group, the benefit was significant by items and marginally significant by participants $\left[F_{1}(1,22)=3.9, p=.062 ; F_{2}(1,190)=7.7, p<.01\right]$.

To examine the cost, we conducted a two-way mixed factorial ANOVA (by participants and by items) with factors of group and study condition (unstudied vs. lure). The main effect for group was significant $\left[F_{1}(1,22)=\right.$ $\left.4.8, p<.05 ; F_{2}(1,190)=9.4, p<.01\right]$. A performance cost was indicated by lower accuracy for lure words (.53) than for unstudied words $(.62)\left[F_{1}(1,22)=11.2, p<.01\right.$; $\left.F_{2}(1,190)=22.8, p<.001\right]$. An interaction between group and study condition $\left[F_{1}(1,22)=9.1, p<.01 ; F_{2}(1,190)=\right.$ $16.8, p<.001]$ indicated that the cost was larger in the control group (.18) than in the amnesic group (.01). An analysis of simple effects indicated that the cost was significant in the control group $\left[F_{1}(1,22)=20.3, p<.001\right.$; $\left.F_{2}(1,190)=39.4, p<.001\right]$, but not in the amnesic group $\left[F_{1}(1,22)=0.06, p>.50 ; F_{2}(1,190)=0.23, p>.50\right]$. We performed a power analysis (Howell, 1992) to determine the power of our measure to detect a performance cost in amnesia, using the benefit of .09 in amnesia to estimate the expected effect size for the cost. Our analysis indicated that the power of our test to detect a cost of this size in the amnesic group was .85 (one-tailed test, $\alpha=.05$ ).

In sum, for high-frequency words, the performance benefit was larger in the control than in the amnesic group but was significant in both groups. The performance cost was significant in the control group, but not in the amnesic group.

\section{Patterns in FCPI With Performance Equated in the Unstudied (Baseline) Condition}

We noted earlier the importance of equating performance in the unstudied condition across groups and conditions in order to examine study-induced performance changes. Because there was a significant effect of word frequency in the unstudied condition and a numerical (albeit nonsignificant) difference between groups in the unstudied condition, we reanalyzed the data with a subgroup of participants in whom performance across these conditions was more closely equated. We excluded the 2 amnesic patients with the highest performance in the low-frequency unstudied condition and the 2 control participants with the lowest performance in the high-frequency unstudied condition. In the resulting subgroup of control participants, accuracy in the unstudied condition was .67 for low-frequency words and .65 for high-frequency words, and in the amnesic subgroup, it was . 70 for low-frequency words and .66 for high-frequency words. These data were submitted to a two-way mixed factorial ANOVA, which revealed no effect of group $(p>.60)$, no effect of word frequency $(p>10)$, and no group $\times$ frequency interaction $(p>.50)$.

The pattern of performance in the FCPI task in this baseline-equated subgroup was essentially the same as that in the whole group, with the exception that some of the effects that were significant in the whole group were at a trend level in the subgroup. In short, with low-frequency words, the benefit was significant in the control group (.87 old vs. .67 unstudied; $p<.001$ ) and in the amnesic group (.81 old vs. .70 unstudied; $p<.05$ ), and the cost was significant in the control group (.67 unstudied vs. .46 lure; $p<.001)$ and marginally significant in the amnesic group (.70 unstudied vs. .62 lure; $p=.061$ ). With highfrequency words, the benefit was significant in the control group (.86 old vs. .65 unstudied; $p<.001$ ) and showed a trend toward significance in the amnesic group (.73 old vs. .66 unstudied; $p=.097)$, and the cost was significant in the control group (.65 unstudied vs. 44 lure; $p<.001$ ) but not in the amnesic group (.66 unstudied vs. .63 lure; $p>.40$ ). Thus, it appears that the pattern that we observed in the group as a whole was not an artifact of differences across groups or conditions in baseline performance. 


\section{Recognition Memory Task}

For the two-choice recognition memory tasks, we calculated, for each participant, in each word-frequency condition, the percentage of words recognized correctly. As was expected, recognition memory was impaired in the amnesic group, both for low-frequency words [amnesic mean $=75.3 \%$, control mean $=96.0 \% ; t(22)=5.5, p<$ .001 ] and for high-frequency words [amnesic mean $=$ $71.8 \%$, control mean $=93.5 \% ; t(22)=6.3, p<.001]$. In the amnesic group, recognition memory was above chance, for both low-frequency words $[t(11)=6.8, p<$ $.001]$ and high-frequency words $[t(11)=6.6, p<.001]$; the difference in recognition accuracy for high- and lowfrequency words did not reach significance $(p>.15)$.

\section{Discussion}

Control participants showed significant costs and benefits in FCPI, with both low-frequency and high-frequency words. The amnesic group showed costs and benefits in FCPI with low-frequency words but failed to show this pattern with high-frequency words. Rather, with highfrequency words, they showed a performance benefit but no performance cost, a pattern consistent with a sensitivity mechanism.

These findings are inconsistent with the argument that our prior finding of a sensitivity pattern in amnesia in FCPI (Keane et al., 2000) was due to the fact that amnesic patients experienced the stimuli as low-frequency words. On that view, the amnesic group in the present study should have shown benefits alone with low-frequency words and a cost/benefit pattern with high-frequency words. Instead, they showed the opposite pattern, with low-frequency words eliciting costs and benefits and high-frequency words eliciting benefits alone.

Two separate aspects of the present findings merit consideration. The first concerns differences in performance between the control and amnesic groups and the implications of those differences for an understanding of the mechanisms underlying cost/benefit patterns of performance in FCPI in control participants. The second concerns the distinct patterns of performance in amnesic participants with high- and low-frequency words and the insights these findings might offer regarding the existence of distinct implicit memory mechanisms. We will consider each of these issues in turn.

\section{Performance Costs and Benefits in FCPI: Mechanisms Underlying Performance in Control Participants}

Control participants consistently show symmetrical costs and benefits in the FCPI paradigm (Keane et al., 2000; Ratcliff \& McKoon, 1997; Ratcliff et al., 1989). In our prior study, because we failed to obtain this pattern in amnesic participants in an FCPI task with word lists of mixed frequency (Keane et al., 2000, Experiment 1), we argued that it was the product of explicit memory mechanisms in control participants. In short, because the identification task is rather difficult, control participants may resort to an explicit memory strategy on many tri- als, opting for the easier task of selecting the response choice corresponding to the studied word. Such a strategy would tend to produce correct responses on old trials (in which the flashed word was indeed the studied word) and incorrect responses on lure trials (in which the flashed word was the mate of the studied word), resulting in performance benefits and costs. We suggested that in control participants, this pattern reflects, at least in part, the operation of explicit memory mechanisms that are unavailable to amnesic patients.

The results of the present study resemble those of our prior study (Keane et al., 2000) in that the performance of the control and amnesic participants differed; the nature of those group differences varied, however, depending on the frequency of the stimuli. In the present study, with high-frequency words, the amnesic patients failed to show the cost/benefit pattern that was observed in the control group and, instead, showed benefits alone in FCPI. This finding is an exact replication of the prior results we obtained in amnesic participants using word lists of mixed frequency (Keane et al., 2000). The present result is consistent with the notion that the cost/benefit pattern with high-frequency words in control participants is mediated, at least in part, by explicit memory processes that are impaired in amnesia.

With low-frequency words in the present study, both groups showed costs and benefits, but these effects were smaller in the amnesic than in the control group. These results suggest that implicit memory mechanisms that are intact in amnesia produce costs and benefits in FCPI with low-frequency words, but that explicit memory mechanisms that are impaired in amnesia contribute to the benefits and costs observed in the control group.

In sum, with regard to the mechanisms mediating performance in the control group, the present results suggest that the cost/benefit patterns with high-frequency and low-frequency words in FCPI are mediated, at least in part, by explicit memory processes that are impaired in amnesia. We now will turn our discussion to the more novel aspect of the present findings, which concerns the different patterns of performance in the amnesic group with high- and low-frequency words.

\section{Distinct Implicit Memory Mechanisms in the FCPI Task}

Because explicit memory is impaired in amnesic individuals, their task performance provides a window onto the operation of implicit memory processes in relative isolation. We therefore will consider how their performance on the present task elucidates the nature of distinct implicit memory mechanisms.

The present results in amnesia were not predicted by either of the theoretical views that motivated the study. The word frequency account (Masson, 2002) of our prior findings in amnesia predicted benefits without costs in amnesia with low-frequency words and a cost/benefit pattern with high-frequency words, whereas our own view predicted benefits without costs with both highand low-frequency words in amnesia. Contrary to both 
views, amnesic patients showed benefits without costs with high-frequency words and a cost/benefit pattern with low-frequency words. One way to understand these results is to consider what is already known about differences in priming effects for high- and low-frequency words.

In standard perceptual identification tasks in which words are flashed in a test phase and no response alternatives are provided, low-frequency words elicit larger priming effects than do high-frequency words (i.e., the difference in identification accuracy for studied and unstudied items is greater for low- than for high-frequency words; Jacoby \& Dallas, 1981). Assuming that these priming effects reflect differential processing fluency for studied and unstudied words, it follows that the fluency differential, or contrast, between studied and unstudied words is greater for low- than for high-frequency words. In the FCPI task used in the present study, studied and unstudied response alternatives were provided after the target word was flashed; the fluency contrast between these response alternatives would have been greater in the low- than in the high-frequency condition. We hypothesize that the magnitude of that fluency differential between the response choices may be great enough in the low-frequency condition (but not in the high-frequency condition) to drive the response choice. As a result, in the low-frequency condition, the more fluent (i.e., studied) response alternative tends to be chosen, resulting in performance benefits and costs. The idea that performance in FCPI can be driven by the fluency differential of response choices (rather than by processing of the flashed word) is consistent with Bowers's (1999) demonstration (discussed earlier) that costs and benefits in FCPI can be obtained even when the flashed "word" is a nonsense string. In the present context, we are suggesting that this fluency-based effect is more likely to occur when the fluency contrast between response alternatives is maximal (i.e., when the words are low frequency). We suggest further that the presence of this effect does not exclude a role for sensitivity in performance, but that the effect of sensitivity (i.e., better discriminability for studied than for unstudied words) may be masked by the stronger influence of the fluency contrast between the response alternatives.

Because the fluency contrast between the response alternatives is not as great in the high-frequency condition, it may have little influence on performance. In this case, we hypothesize, performance is driven not by the fluency differential of the response choices, but by the discriminability of flashed words (i.e., by the participant's perceptual sensitivity to flashed words): Words that have appeared in the prior study phase are more accurately identified than words that have not (i.e., unstudied and lure words), so the correct response alternative is chosen more often in the old condition than in the unstudied or lure condition, and accuracy does not differ in the latter two conditions. Thus, in the high-frequency condition, the operation of a sensitivity mechanism is apparent and is unobscured by the relatively subtle fluency differential between the response alternatives.

In sum, we hypothesize that in amnesic participants, performance in the FCPI task is driven by the study- induced fluency contrast between response alternatives in the low-frequency condition and by study-induced sensitivity changes operating on the flashed word in the high-frequency condition. Before testing this hypothesis, however, we will consider the possibility that performance in amnesia can be understood in terms of weak residual explicit memory capacities coupled with a sensitivity mechanism. On this view, only one additional mechanism beyond explicit memory (i.e., a sensitivity mechanism) is required to explain performance across amnesic and control participants in this paradigm. Because explicit memory is above chance (but below normal) in the amnesic group, it is possible that this residual capacity produced the (above-chance but below-normal) costs and benefits in the low-frequency condition in amnesia. By this view, the absence of a cost/benefit pattern for high-frequency words could be due to the fact that explicit memory (which is numerically, albeit not statistically, lower for high- than for low-frequency words in amnesia) is insufficient to support costs/benefits in that condition. Correlational analyses, however, did not provide support for this view. In the low-frequency condition in amnesia, there was no association between explicit memory and benefit scores (old minus unstudied), between explicit memory and cost scores (unstudied minus lure), or between explicit memory and combined cost/benefit scores (old minus lure) (all $p \mathrm{~s}>.50)$.

Thus, we entertain the hypothesis that performance in amnesia in the FCPI task is driven by a fluency-based response choice mechanism for low-frequency words and by a sensitivity mechanism for high-frequency words. We tested this hypothesis in Experiment 1B by examining the performance of amnesic patients in an FCPI task that differed in only one respect from the task in Experiment 1A: In the test phase, the participants were told that a word would be flashed and followed by two response alternatives, but in reality, no test word was flashed. Instead, a blank screen followed by a 500 -msec mask was presented on each test trial and was followed by two response alternatives (a studied word and its orthographic mate), as in Experiment 1A. Old, unstudied, and lure trials (and what was considered the accurate response on each trial) were defined on the basis of the stimulus that would have been flashed on each trial in Experiment 1A. In reality, however, old and lure trials were indistinguishable from each other in Experiment 1B: On both sorts of trials, a mask was flashed and was followed by two response alternatives, including a studied word and its unstudied orthographic mate. (In the unstudied condition, the response alternatives included a word and its orthographic mate, but in this case, both response alternatives were unstudied.) Thus, in Experiment 1B, because there was no actual stimulus to identify in the test phase, the response could be driven only by the characteristics of the two response alternatives (and performance in the unstudied condition would be expected to be at chance). If the participants tended to choose the studied response on old and lure trials, they should show "benefits" (choosing the studiedi.e., "correct" - alternative in the old condition more often than in the unstudied condition), and "costs" (choosing 
the studied-i.e., "incorrect"- alternative in the lure condition more often than in the unstudied condition).

Following our account of the results in amnesia from Experiment $1 \mathrm{~A}$, we made the strong prediction that, for low-frequency words in Experiment 1B, there would be a significant benefit and cost, as there was in Experiment 1A. This prediction follows from the hypothesis that the costs and benefits in amnesia for low-frequency words in Experiment 1A were driven by the study-induced fluency contrast between the response alternatives in the test phase. Because the absence of a flashed word in the test phase in Experiment 1B would not affect that fluency differential, we expected to observe similar costs and benefits under these conditions.

The predictions for high-frequency words in Experiment $1 \mathrm{~B}$ were less clear, because the presence of benefits without costs for these stimuli in Experiment 1A was presumably driven not by the fluency differential between the response alternatives, but by the identifiability of flashed words in the test phase (as a function of whether or not they had been encountered in the study phase). Because the flashed word was absent in Experiment 1B, we expected, at a minimum, not to observe a benefit in the absence of a cost. It was possible, however, that in the absence of the opportunity for perceptual sensitivity effects to influence performance, we would observe modest costs and benefits for high-frequency words in Experiment 1B. Such a pattern would presumably reflect the subtle fluency contrast between the studied and unstudied response alternatives, even in the high-frequency condition.

In sum, Experiment 1B was designed to test a hypothesis about two distinct implicit memory processes that contributed to the performance of the amnesic patients in FCPI with high- and low-frequency words in Experiment 1A. Because the same pattern of performance was not observed in the control participants (presumably, because performance in that group was mediated by explicit memory processes), and because the performance of amnesic patients provides a purer measure of implicit memory processes, Experiment $1 \mathrm{~B}$ included only amnesic participants.

\section{EXPERIMENT 1B}

\section{Method}

\section{Participants}

This experiment included 16 amnesic individuals, 11 of whom had participated in Experiment 1A. (There was an interval of at least 3 months between the two experiments.) This group consisted of 7 men and 9 women with amnesias of varying etiologies, including anoxia ( $n=6$, one with a left partial temporal lobectomy), encephalitis $(n=3)$, bithalamic stroke $(n=1)$, and anterior communicating artery aneurysm $(n=6)$. They had a mean age of 59.3 years, a mean education of 13.9 years, and a mean WAIS-III verbal IQ score of 101.1. Their attentional abilities were intact, as indicated by a mean WMS-III Working Memory Index of 96.1. Their memory functioning was severely impaired, as indicated by a mean General Memory Index of 61.9, a mean Visual Delay Index of 68.3, and a mean Auditory Delay Index of 62.5 (see Table 2).

\section{Materials, Design, and Procedure}

The materials, design, and procedure were identical to those used in Experiment 1A, with the following exceptions: (1) In the test phase of the perceptual identification task, a 16-msec blank screen was flashed, instead of a word, and was followed by a $500-\mathrm{msec}$ mask of superimposed $\mathrm{H}$ and X characters; (2) because no words were flashed in the test phase of the priming task, there was no preexperimental setting of stimulus duration; and (3) there was no recognition memory task.

The two priming tasks (one with high- and one with low-frequency words) were administered in one session, with the order of highand low-frequency conditions counterbalanced across participants. Each participant completed the experiment twice, with alternate test forms, in sessions separated by at least 3 weeks. (For 3 participants, technical problems prevented completion of one of the priming tasks in one session. In those cases, results from the other session were used in place of a mean across sessions.)

\section{Results}

\section{Low-Frequency Words}

For each participant, we calculated the proportion of low-frequency words identified "correctly" (i.e., in accord with what would have been the correct response had a test word been flashed) in each experimental condition (old, unstudied, lure; see Table 4). These data were submitted to a one-way ANOVA (both by participants and by items) followed by planned contrasts to examine the benefit (old vs. new) and the cost (new vs. lure). There was a significant effect for condition $\left[F_{1}(2,30)=5.6, p<\right.$ $\left..01 ; F_{2}(2,190)=18.3, p<.001\right]$. A benefit, indicated by higher accuracy in the old than in the unstudied condition, was significant $\left[F_{1}(1,15)=5.3, p<.05 ; F_{2}(1,95)=13.2\right.$, $p<.001]$. A cost, indicated by a reduction in performance in the lure, as compared with the unstudied, condition, was also significant $\left[F_{1}(1,15)=5.0, p<.05 ; F_{2}(1,95)=\right.$ $8.6, p<.01]$.

\section{High-Frequency Words}

For each participant, we calculated the proportion of high-frequency words identified "correctly" in each experimental condition (Table 4). A one-way ANOVA indicated that there was a significant effect of condition only in the item analysis $\left[F_{1}(2,30)=1.8, p>.15\right.$; $\left.F_{2}(2,190)=10.0, p<.001\right]$. Planned contrasts for the item analysis indicated that that there was a significant benefit $\left[F_{2}(1,95)=7.1, p<.01\right]$ and a significant cost $\left[F_{2}(1,95)=4.8, p<.05\right]$.

\section{Discussion}

The goal of Experiment 1B was to test a hypothesis about two distinct implicit memory processes that may contribute to performance in amnesia in the FCPI task: (1) a sensitivity mechanism that facilitates identification

Table 4

Mean Proportion Correct in Forced Choice Perceptual Identification Task With Flashed Word Omitted at Test (Experiment 1B; $n=16$ Amnesic Participants)

\begin{tabular}{|c|c|c|c|c|c|c|}
\hline \multirow[b]{3}{*}{ Frequency } & \multicolumn{6}{|c|}{ Condition } \\
\hline & \multicolumn{2}{|c|}{ Old } & \multicolumn{2}{|c|}{ Unstudied } & \multicolumn{2}{|c|}{ Lure } \\
\hline & $M$ & $S E$ & $M$ & $S E$ & $M$ & $S E$ \\
\hline Low & .63 & .05 & .52 & .02 & .43 & .04 \\
\hline High & .56 & .05 & .50 & .02 & .44 & .04 \\
\hline
\end{tabular}


of studied stimuli in the test phase, yielding benefits in the absence of costs (as observed with high-frequency words in the amnesic group in Experiment 1A), and (2) a fluencybased response choice mechanism that drives selection of a response choice in the test phase, yielding a pattern of benefits and costs (as observed with low-frequency words in the amnesic group in Experiment 1A). Experiment 1B was designed to eliminate the possible contribution of the first (sensitivity) mechanism to performance in the FCPI task by omitting the flashed word from the test phase, leaving only the fluency contrast between the response choices to drive performance. For low-frequency words, we predicted that, despite the omission of the flashed word, we would still observe benefits and costs. The results are consistent with this prediction. For high-frequency words, we predicted that the omission of the flashed word would eliminate the sensitivity pattern (a benefit in the absence of a cost) that was observed in the amnesic group in Experiment $1 \mathrm{~A}$. Our findings are consistent with this prediction in that they revealed (in the item analysis) a cost/ benefit pattern in place of a benefit alone. Thus, in the absence of the possibility for a sensitivity mechanism to influence performance, a response choice fluency pattern emerged for high-frequency words.

\section{GENERAL DISCUSSION}

The present study revealed an unexpected role for word frequency in the performance of amnesic patients in the FCPI task: With low-frequency words, there were benefits and costs in performance, whereas with high-frequency words, there was a benefit alone in performance (Experiment 1A). For low-frequency words, the benefit/cost pattern was present even when the flashed word was omitted in the test phase (Experiment 1B), consistent with the idea that this effect operated at the level of response choices and that it resulted from the participants' tendency to choose the alternative that was more fluent. For high-frequency words, the sensitivity effect was eliminated by omitting the flashed word (Experiment 1B), consistent with the notion that that this effect in Experiment 1A operated at the level of identification of the flashed word.

\section{Implicit Memory Mechanisms Mediating Performance on the FCPI Task}

Our results demonstrate that, when the contribution of explicit memory performance is reduced or eliminated (as in amnesia), the operation of two mechanisms becomes apparent in the FCPI task: a fluency-based response choice mechanism (yielding costs and benefits in performance) and a sensitivity mechanism (yielding benefits in the absence of costs).

The fact that the benefit/cost pattern was present even under conditions in which no test word was flashed (Experiment 1B) is theoretically important: This effect cannot be explained by Ratcliff and McKoon's (1997) counter model, which assumes that such effects reflect biased processing of the flashed word. This finding suggests, instead, that the cost/benefit pattern in the FCPI paradigm reflects, at least in part, processes operating at the level of the response choices (see also Bowers, 1999).

Although we did not observe sensitivity effects for low-frequency words in the present study, we do not infer thereby that sensitivity effects cannot be obtained for such stimuli. Indeed, a number of studies have demonstrated sensitivity effects for low-frequency words in a version of the FCPI task that differs slightly from the one used in the present study (see below). We suggest that in the present paradigm, sensitivity effects in amnesia for low-frequency words may have been masked by the more potent fluencybased response choice effect.

Our finding in amnesia of sensitivity effects in FCPI with high-frequency words presents something of a puzzle, since it appears to be at odds with several published findings in normal cognition that suggest that sensitivity effects are not obtained for high-frequency words in the FCPI task (McKoon \& Ratcliff, 2001; Wagenmakers, Zeelenberg, \& Raaijmakers, 2000). There is one methodological difference between those studies and the present one: In most prior studies, sensitivity has been measured as the difference in identification accuracy in a condition in which both response choices (e.g., case and care) appeared in the study phase, as compared with a baseline condition in which neither response choice appeared in the study phase (equivalent to our unstudied condition). It has been argued that any performance improvement in the both-studied, as compared with the neither-studied, condition could not be due to bias, because a study-induced bias would favor equally both response choices in the bothstudied condition. Such an effect could be due only to enhanced sensitivity for flashed words that had appeared in the prior study phase. ${ }^{1}$ In normal cognition, this sensitivity effect has been observed for low-frequency words but has not been observed for high-frequency words. Because the present paradigm did not include a both-studied condition, it provided a different measure of sensitivity: a performance benefit (old $>$ unstudied) in the absence of a performance cost (i.e., no decrement in the lure, as compared with the unstudied, condition). ${ }^{2}$ By this measure, we observed a sensitivity effect for high-frequency words in amnesia. Future research will be needed to ascertain whether our findings in amnesia are paradigm specific or whether they reflect a fundamental difference in the nature of the mechanisms that mediate priming in amnesia and in normal cognition.

\section{Explicit Memory Contributions to Performance in the FCPI Task}

The distinct patterns of performance for high- and low-frequency words that we observed in amnesia were not evident in the performance of healthy control participants in Experiment 1A; instead, control participants showed costs and benefits (exceeding in magnitude those observed in amnesia) for both high- and low-frequency words. We hypothesize that the FCPI task elicited explicit memory strategies in our control group that were unavailable to the amnesic group (see also Keane et al., 2000). Such strategies may have led the participants, on many tri- 
als, to select the response choice that had appeared in the prior study phase. Because the studied choice is correct on old trials and incorrect on lure trials, such an approach would produce both benefits (old $>$ unstudied) and costs (lure $<$ unstudied) in performance. By this view, the performance of the control participants in the present study does not provide a window onto the nature of the implicit memory processes that may mediate performance in the FCPI task.

These findings (and similar results in Keane et al., 2000, Experiment 1) highlight the need for caution when interpreting the performance of control participants in the FCPI task. Findings from control participants in this paradigm have been central to theories about the cognitive mechanisms underlying implicit memory (e.g., Ratcliff \& McKoon, 1997; Schooler, Shiffrin, \& Raaijmakers, 2001). It is therefore important to be sure that the performance of control participants does indeed reflect the operation of implicit, rather than explicit, memory mechanisms. Our findings in amnesia suggest that, at least under some conditions, the performance of control participants reflects the operation of explicit memory strategies. McKoon and Ratcliff (2001) pointed out that the nature of the instructions in the study phase may influence the likelihood that control participants will use explicit memory in the identification task; for example, when instructed to memorize the words in the study phase for a later memory test, control participants will be less likely to use explicit memory in the identification task because they assume that another (explicit) test phase will follow. McKoon and Ratcliff (2001) suggested that when the study phase is not assigned a purpose (e.g., when participants are asked simply to read words aloud), they are more likely to assume that it is intended to aid them in the subsequent identification task and so will think back to the studied words (i.e., will use explicit memory) when performing the identification task. The present findings suggest that even when participants are assigned a task to complete in the study phase (in this case, to rate how positive or negative words are), they may still use explicit memory in the identification task.

\section{Summary and Conclusions}

The present study explored the role of word frequency in study-induced performance changes in an FCPI task. Control participants showed performance costs and benefits in FCPI with high- and low-frequency words, replicating well-established effects from prior studies in normal cognition that used mixed-frequency lists. The pattern of performance in the amnesic group differed from that in the control group, consistent with the idea that the cost/ benefit effects in FCPI observed in normal cognition reflect a contribution of explicit memory processes that are not available in amnesia (see also Keane et al., 2000). The results from the amnesic group provide a more accurate index of implicit memory processes in FCPI, and those results revealed a cost/benefit pattern in FCPI with low-frequency words and benefits alone in FCPI with high-frequency words. Omission of the flashed word in the test phase eliminated the benefits-only pattern with high-frequency words in amnesia but did not eliminate the cost/benefit pattern with low-frequency words in amnesia, consistent with the idea that the cost/benefit pattern reflects the differential fluency of the response choices (rather than biased processing of the flashed word). These results replicate and extend our prior findings in amnesia, which demonstrated sensitivity effects in an FCPI task with mixed-frequency word lists (Keane et al., 2000). Together with our prior findings, the present results in amnesia suggest that implicit memory effects in the FCPI task reflect the operation of at least two mechanisms - a fluency-based response choice mechanism and a perceptual sensitivity mechanism - the relative contributions of which may vary with the frequency of the test stimuli. These findings are in keeping with the spirit of recent empirical and modeling work that has highlighted the combined contributions of multiple mechanisms to a broad range of priming effects in normal cognition (Ratcliff \& McKoon, 2000; Zeelenberg et al., 2002).

\section{AUTHOR NOTE}

This work was supported by NIMH Grants MH70830 and MH57681 and by the Medical Research Service of the United States Department of Veterans Affairs. We thank Karen Fossum for assistance with data analyses. We are grateful to Barbara Church, John Dunn, and an anonymous reviewer for helpful comments on the manuscript. Correspondence concerning this article should be addressed to M. M. Keane, Department of Psychology, Wellesley College, Wellesley, MA 02481 (e-mail: mkeane@) wellesley.edu).

\section{REFERENCES}

Blaxton, T. A. (1989). Investigating dissociations among memory measures: Support for a transfer-appropriate processing framework. Journal of Experimental Psychology: Learning, Memory, \& Cognition, 15, 657-668. doi:10.1037/0278-7393.15.4.657

Bowers, J. S. (1999). Priming is not all bias: Commentary on Ratcliff and McKoon (1997). Psychological Review, 106, 582-596. doi:10.1037/0033-295X.106.3.582

Bowers, J. S., \& KouIDER, S. (2003). Developing theories of priming with an eye on function. In J. S. Bowers \& C. J. Marsolek (Eds.), Rethinking implicit memory (pp. 19-40). Oxford: Oxford University Press.

Chapman, L. J., Chapman, J. P., Curran, T. E., \& Miller, M. B. (1994)

Do children and the elderly show heightened semantic priming? How to answer the question. Developmental Review, 14, 159-185.

FrancIs, W. N., \& KuČERA, H. (1982). Frequency analysis of English usage: Lexicon and grammar. Boston: Houghton Mifflin.

Graf, P., \& Ryan, L. (1990). Transfer-appropriate processing for implicit and explicit memory. Journal of Experimental Psychology: Learning, Memory, \& Cognition, 16, 978-992. doi:10.1037/0278 $-7393.16 .6 .978$

Howell, D. C. (1992). Statistical methods for psychology. Belmont, CA: Wadsworth.

JACOBY, L. L., \& Dallas, M. (1981). On the relationship between autobiographical memory and perceptual learning. Journal of Experimental Psychology: General, 110, 306-340. doi:10.1037/0096 $-3445.110 .3 .306$

Keane, M. M., Verfaellie, M., Gabrieli, J. D. E., \& Wong, B. M. (2000). Bias effects in perceptual identification: A neuropsychological investigation of the role of explicit memory. Journal of Memory \& Language, 43, 316-334. doi:10.1006/jmla.2000.2732

Masson, M. E. J. (2002). Bias in masked word identification: Unconscious influences of repetition priming. Psychonomic Bulletin \& Review, 9, 773-779.

McKoon, G., \& Ratcliff, R. (2001). Counter model for word identification: Reply to Bowers (1999). Psychological Review, 108, 674-681. doi:10.1037/0033-295X.108.3.674

Moscovitch, M., Goshen-Gottstein, Y., \& Vriezen, E. (1994). Memory without conscious recollection: A tutorial review from a 
neuropsychological perspective. In C. Umiltà \& M. Moscovitch (Eds.), Attention and performance $X V$ : Conscious and nonconscious information processing (pp. 619-660). Cambridge, MA: MIT Press, Bradford Books.

RAtCliff, R., Allbritton, D., \& McKoon, G. (1997). Bias in auditory priming. Journal of Experimental Psychology: Learning, Memory, \& Cognition, 23, 143-152. doi:10.1037/0278-7393.23.1.143

RatclifF, R., \& McKoon, G. (1996). Bias effects in implicit memory tasks. Journal of Experimental Psychology: General, 125, 403-421. doi:10.1037/0096-3445.125.4.403

RATCLIFF, R., \& McKoon, G. (1997). A counter model for implicit priming in perceptual word identification. Psychological Review, 104, 319-343. doi:10.1037/0033-295X.104.2.319

Ratcliff, R., \& McKoon, G. (2000). Modeling the effects of repetition and word frequency in perceptual identification. Psychonomic Bulletin \& Review, 7, 713-717.

Ratcliff, R., McKoon, G., \& Verwoerd, M. (1989). A bias interpretation of facilitation in perceptual identification. Journal of Experimental Psychology: Learning, Memory, \& Cognition, 15, 378-387. doi:10.1037/0278-7393.15.3.378

Reber, R., Winkielman, P., \& Schwartz, N. (1998). Effects of perceptual fluency on affective judgments. Psychological Science, 9, 45-48. doi:10.1111/1467-9280.00008

Roediger, H. L., III (2003). Reconsidering implicit memory. In J. S. Bowers \& C. J. Marsolek (Eds.), Rethinking implicit memory (pp. 3-18). Oxford: Oxford University Press.

RoEDiger, H. L., III, \& BlaXton, T. A. (1987). Retrieval modes produce dissociations in memory for surface information. In D. S. Gorfein \& R. R. Hoffman (Eds.), Memory and learning: The Ebbinghaus centennial conference (pp. 349-379). Hillsdale, NJ: Erlbaum.

Roediger, H. L., III, \& McDermott, K. B. (1993). Implicit memory in normal human subjects. In F. Boller \& J. Grafman (Eds.), Handbook of neuropsychology (Vol. 8, pp. 63-131). Amsterdam: Elsevier.

SCHACTER, D. L. (1990). Perceptual representation systems and implicit memory: Toward a resolution of the multiple memory systems debate. In A. Diamond (Ed.), The development and neural bases of higher cognitive functions (Annals of the New York Academy of Sciences, Vol. 608, pp. 543-571). New York: New York Academy of Sciences. doi:10.1111/j.1749-6632.1990.tb48909.x

SCHaCter, D. L. (1992). Priming and multiple memory systems: Perceptual mechanisms of implicit memory. Journal of Cognitive Neuroscience, 4, 244-256. doi:10.1162/jocn.1992.4.3.244

Schooler, L. J., Shiffrin, R. M., \& RaAijmakers, J. G. W. (2001). A Bayesian model for implicit effects in perceptual identification. Psychological Review, 108, 257-272. doi:10.1037/0033-295X .108 .1 .257

SeAmon, J. G., Brody, N., \& KaUfF, D. M. (1983). Affective discrimination of stimuli that are not recognized: II. Effect of delay between study and test. Bulletin of the Psychonomic Society, 21, 187-189.

Tulving, E., \& Schacter, D. L. (1990). Priming and human memory systems. Science, 247, 301-306. doi:10.1126/science.2296719

Verfaellie, M., \& Keane, M. M. (2001). Scope and limits of implicit memory in amnesia. In B. De Gelder, E. H. F. De Haan, \& C. A. Heywood (Eds.), Out of mind: Varieties of unconscious processes (pp. 151-162). Oxford: Oxford University Press.

WAGEnMaKers, E.-J. M., Zeelenberg, R., \& RaAiJmakers, J. G. W. (2000). Testing the counter model for perceptual identification: Effects of repetition priming and word frequency. Psychonomic Bulletin \& Review, 7, 662-667.

Wagenmakers, E.-J. M., Zeelenberg, R., Schooler, L. J., \& RaAiJMAKERS, J. G. W. (2000). A criterion-shift model for enhanced discriminability in perceptual identification: A note on the counter model. Psychonomic Bulletin \& Review, 7, 718-726.

WeChSLER, D. (1997a). Wechsler Adult Intelligence Scale-III. San Antonio, TX: Psychological Corporation.

WeChSLER, D. (1997b). Wechsler Memory Scale-III. San Antonio, TX: Psychological Corporation.

Whittlesea, B. W. A., JACOBY, L. L., \& Girard, K. (1990). Illusions of immediate memory: Evidence of an attributional basis for feelings of familiarity and perceptual quality. Journal of Memory \& Language, 29, 716-732. doi:10.1016/0749-596X(90)90045-2

Zeelenberg, R., Wagenmakers, E.-J. M., \& Raaijmakers, J. G. W. (2002). Priming in implicit memory tasks: Prior study causes enhanced discriminability, not only bias. Journal of Experimental Psychology: General, 131, 38-47. doi:10.1037/0096-3445.131.1.38

\section{NOTES}

1. Note, however, that Raaijmakers and colleagues have argued that such findings can be accommodated by models that assume a shift in bias with no change in sensitivity (Schooler, Shiffrin, \& Raaijmakers, 2001; Wagenmakers, Zeelenberg, Schooler, \& Raaijmakers, 2000).

2. Depending on how a change in sensitivity is operationalized, such a change may or may not entail a performance cost. For example, according to the probabilistic model for bias and sensitivity changes described by Ratcliff et al. (1989), a cost in the FCPI task occurs only with a change in bias and is not associated with a change in sensitivity. Ratcliff and McKoon (1996) have argued that "an increased facility [for detecting stimuli] would not predict that there would sometimes be costs to performance" (p. 414). On the other hand, Bowers and Kouider (2003) have argued that "learning systems that improve processing do not rule out costs in performance, although the benefits outweigh the costs in the domain in which they function" (p. 32). On this view, the presence of costs does not rule out a pure sensitivity mechanism (as long as those costs are smaller than the accompanying benefits). For explicatory purposes in the present article, we have adopted a framework in which performance costs are uniquely associated with a bias mechanism. Our interpretation of the present results does not hinge on this assumption, however, because all views agree that a cost and benefit of equal magnitude indicates the operation of bias, whereas a benefit in the absence of a cost indicates a change in sensitivity.

(Manuscript received September 10, 2007; revision accepted for publication February 5, 2009.) 